\title{
Pathophysiological effect of hypernatremia in relation to the home measurement of blood Pressure (Mdpa) in high andean people. Ayacucho - peru
}

\begin{abstract}
The present research work had the following objectives: to know the pathophysiological effect of hypernatremia in relation to blood pressure measurement (MDPA) in high Andean settlers. To determine the factors that generate hypernatremia in relation to blood pressure measurement (MDPA) in high Andean settlers.

The applied method was Barochiner's based on home blood pressure measurement (MDPA), it also offers advantages over other ambulatory blood pressure (BP) measurement methods, such as greater acceptance by patients, lower cost and Treatment was monitored in some rural communities such as Huascahura, Rancha, Socos, Vinchos, Quinua, Acosvinchos, Acocro and Mollepata.

Among the results obtained we have the following: the pathophysiological effect of hypernatremia in relation to the measurement of blood pressure (MDPA) in high Andean settlers of $36 \%$ The prevalence of $80 \%$, in relation to the measurement of blood pressure (MDPA) in settlers high Andes. The factors that generate hypernatremia in relation to blood pressure measurement (MDPA) in high Andean settlers such as low consumption of beverages, dehydration, poor diet, pathologies and self-medication. Finally, the reproducibility and reliability of the MDPA protocol has been determined using Cronbach's alpha equivalent to 0.87 .
\end{abstract}

Keywords: hyponthermia, hypernatremia blood pressure, table salt
Volume |4 Issue 6 - 202|

\author{
Jesús Javier Ñaccha Urbano \\ Departmet of Biology and Veterinary Medicine, San Cristóbal of \\ Huamanga University, Perú
}

\begin{abstract}
Correspondence: Jesús Javier Ñaccha Urbano, Biology and Veterinary medicine, Departmet of Biology and Veterinary Medicine, San Cristóbal of Huamanga University, Perú, Email jesus.naccha@unsch.edu.pe
\end{abstract}

Received: May 25, 2021 | Published: November 19, 2021

\section{Introduction}

The present research work entitled: Physiopathological effect of hypernatremia in relation to the measurement of blood pressure (MDPA) in high Andean inhabitants of the Ayacucho region, prior voluntary consent, was carried out from the survey on their diet and blood pressure as taken from samples "in situ" in some high Andean communities such as Huascahura, Rancha, Socos, Vinchos, Quinua, Acosvinchos, Acocro among others, data processing in the Laboratory of Physiology and Natural Sciences of the Faculty of Biological Sciences of the UNSCH.

Sodium chloride $(\mathrm{NaCl})$ composed of approximately $60 \%$ chlorine that provides the salty taste and $40 \%$ sodium. This proportion, salt, constitutes the major source of sodium in the diet of the high Andean population. There is also another part of the sodium that comes directly from food or its manufacturing processes, as well as from additives or preservatives, it is also known as "table salt".

The present work was carried out in the high Andean towns and communities of the province of Huamanga, how much is the daily consumption of salt $/ \mathrm{g}$, when the recommendation of the WHO is not to exceed $5 \mathrm{~g} /$ day (the salt that fits in a thimble) equivalent to $2 \mathrm{~g}$ of sodium, preferably iodine. The MDPA also offers advantages over other ambulatory blood pressure measurement methods, such as greater acceptance by patients, lower cost, and long-term treatment follow-up. ${ }^{1}$

Although the many studies that exist in this field have used different measurement protocols, in recent years guidelines have been published for the measurement of home blood pressure that agree to perform at least days of PADD, discarding the first day of measurements for the analysis, since they are considered more unstable., ${ }^{2,3}$

Although in our country recommendations based primarily on the study of hypertension in urban populations (provincial and district capitals) are used, the applicability of these guidelines is not tested in the rural high Andean population.

The high consumption of salt causes cerebrovascular alterations and ischemic heart disease are responsible for more than half of the deaths due to diseases of the circulatory system in urban and rural populations. The determinants of the appearance of these diseases, the cardiovascular risk factors, are multiple and in general, they are common predictors for all western populations where they have been studied. However, of the wide range of known cardiovascular risk factors, probably high blood pressure, smoking, diabetes mellitus, and hypercholesterolemia are of concern to rural populations.

Despite clinical experience, and numerous epidemiological, clinical, and molecular studies that support the direct association between sodium $(\mathrm{Na})$ and blood pressure, discrepancies still persist on the effectiveness and safety of salt restriction to prevent and treat hypertension. ${ }^{4}$

We must consider the high Andean population as a new or inclusive case, linked to a family study, and we are obliged to install preventive measures in their children.

Although there is still no simple intervention to determine the sensitivity or insensitivity to salt, we have to consider that the salt 
intake in the high Andean region of Ayacucho is high, basically of salty cheese, salty dried meat, salty corn and other products made with salt like olives among others.

\section{The objectives set out in this research work were the following}

To know the pathophysiological effect of hypernatremia in relation to blood pressure measurement (MDPA) in high Andean settlers. To determine the factors that generate hypernatremia in relation to blood pressure measurement (MDPA) in high Andean settlers.

\section{Background}

Studies carried out in young individuals under 30years of age, saltsensitive hypertension occurs in $50 \%$ of cases, whereas in subjects over 50years of age, more than $80 \%$ have salt-sensitive hypertension. In addition, with age the sensitivity of pressure to salt ingestion also increases. ${ }^{5}$ Subsequently, it was shown that subjects between 20 and 30 years of age, the change in salt intake produced an increase of 5 mmHg in TAM. ${ }^{6}$

The application of hypertonic solutions in the third ventricle of rats for 7 days, and demonstrated an increase in systolic pressure of $23 \pm 5 \mathrm{~mm} \mathrm{Hg}$ on the 1 st day and $15 \pm 2 \mathrm{~mm} \mathrm{Hg}$ on the 7 th, in addition to higher water intake, hyponatremia, decrease in plasma osmolarity and increase in plasma norepinephrine 37.38.

About $50 \%$ of essential hypertensive patients hypertension is sensitive to salt, this characteristic is accentuated and its frequency increases with age and is associated with an increased risk of cardiovascular complications and nephropathy.5 The responsible mechanism has not been established in precision, although the participation of different vasoactive factors has been suggested. The sensitivity to salt implies an alteration in the relationship between arterial pressure and sodium excretion or "pressure natriuresis" which shifts to the right, that is, a higher arterial pressure is required to maintain normal excretion of salt.

Recently it was observed that chronic infusion of angiotensin II in rats produced severe hypertension and histological changes similar to those described above. After suspending the infusion, blood pressure decreased to normal levels, however the administration of a high-salt diet produced a progressive elevation of $\mathrm{BP}$, that is, hypertension sensitive to salt. These observations suggest that histological alterations play a fundamental role in the generation of arterial hypertension and in salt sensitivity. ${ }^{8}$

On the other hand, in studies carried out in patients with arterial hypertension, in which renal histology was studied, the histological changes are very similar to those observed in the contralateral kidney of rats with renovascular hypertension. ${ }^{9}$

Importance of sodium and volume in the appearance of hypertension. Finding the cause or causes of high blood pressure has been one of the most notorious frustrations in 20th century medicine. Perhaps the most important reason is the patent obstinacy to accept the increase in peripheral vascular resistance (present in all forms of fixed hypertension), as a primary mechanism and not as a predictable biological response to circumstances that require and are resolved by means of vasoconstriction. For this same reason, efforts have been directed at treating what could be a consequence (vasoconstriction) and not a cause.

Undoubtedly, in high blood pressure as in other diseases, multiple factors can facilitate its development (genes, diet, environment and behaviors). This could mean that hypertension is not one but several diseases that are expressed with elevated blood pressure values, or on the contrary, there are many factors that produce a single effect and from which the body defends itself by raising blood pressure.

Precisely the elevation of blood pressure is a maladaptive mechanism by which blood pressure increases to be able to filter and eliminate loads of salt that could eventually translate into much more dangerous cardiovascular congestion. This phenomenon was called "pressure diuresis" and it has been demonstrated in each experimental model and clinical situation in which it has been studied. Furthermore, an adequate natriuretic response is incompatible with the maintenance of the hypertensive picture. ${ }^{7}$

Actually, according to Guyton's theory, the kidney is only the end-effector. For example, a perfectly healthy kidney is not responsible for saline retention when ordered to do so, as occurs in some secondary forms of hypertension (primary hyperaldosteronism, pheochromocytoma, etc.). ${ }^{7}$

The concept that vasoconstriction mechanisms are triggered by a perception (real or erroneous) of excess saline, has been underpinned by countless clinical, epidemiological and experimental studies. Unfortunately, clinicians and specialists assumed that the interpretation of this theory held the kidney responsible in all cases. ${ }^{8}$

The truth is that, if the perceived information (real or erroneous) indicates that there is an extracellular excess (excess saline) that has not been able to be evacuated by normal volume detection mechanisms, the answer will be to raise blood pressure to induce salt excretion in the only organ that can do it efficiently. ${ }^{9}$

Recently, the hypothesis has been proposed that, with an increase in salt intake, the osmolar concentration in the blood would increase and, thus, the vasoconstrictor response to sodium.

The human being has two kidneys with a total weight of $250 \mathrm{~g}$ that receive 1 liter of blood per minute, the highest blood flow/g of tissue in the economy. This enables glomerular filtration, which for a normal adult is 180 liters / day.

This very significant volume in the tubular lumen requires an energy resorption effort that is reasonable in light of the importance of allowing the renal tubules to come into contact with blood volume 36 times per day. In this way, kidney tissue regulates blood volume in less than half an hour, fulfilling its fundamental task: controlling the internal environment.

The magnitude of the glomerular filtration volume and tubular reabsorption requires highly precise regulation mechanisms. Small abnormalities in these mechanisms can produce significant changes in sodium balance.

The existence of several interrelated regulatory pathways in processes that require meticulous and dynamic control is a constant in the different cellular and physiological mechanisms.

Regulation of renal flow, specifically constant glomerular filtration, depends on the response of the afferent arteriole to changes in renal perfusion pressure and the glomerular tubule balance, which relates the volume of glomerular filtration to the tubular fluid that reaches the distal segments. and multiple local and general endocrine systems that regulate vascular tone and intra-renal flow. The different tubular segments have their own anatomical and histological characteristics determined by their physiological functions. ${ }^{10}$

Although we have osmolar receptors that activate the mechanisms of thirst and secretion of antidiuretic hormone, there are no data 
that an increase in osmolarity raises blood pressure directly, or at least increases effective arterial volume. The theory remains in the realm of hypotheses. In any case, salt is a central component in the pathogenesis of arterial hypertension and epidemiological evidence supports it. Indeed, population studies mostly agree that excessive salt intake is associated with a higher prevalence of hypertension, while populations and ethnic groups with low salt intake maintain low blood pressure levels compared to those civilizations with high consumption. ${ }^{11}$

\section{Hormonal factors that affect sodium excretion}

There are several alterations in aldosterone synthesis that cause $\mathrm{Na}$ retention. The most marked clinical form is primary hyperaldosteronism, caused by adrenal adenoma, followed by nodular hyperplasia, diffuse hyperplasia, and hyperaldosteronism inappropriate for the renin level. There are genetic defects that determine an increase in mineralocorticoid action, such as the hybrid gene that gives sensitivity to ACTH to aldosterone synthetase, alterations in 1 ip and 17a hydroxylase that increase levels of deoxycorticosterone, and defects in the synthesis of cortisol due to alteration of 1 ip-hydroxysteroid dehydrogenase that interferes with the passage of cortisol to cortisone, favoring its accumulation and its mineralocorticoid action. ${ }^{12}$

The kallikrein-kinin system, which exerts a natriuretic effect, is expressed in the connecting segment of the distal tubules. Strains deficient in kininogen and animals affecting the bradykinin receptor 2 revealed that the kallikrein-kinin system only induces natriuresis when there is a marked retention of sodium resulting from a high intake or excess of aldosterone. On the contrary, the stimulation of the tubular synthesis of kallikrein by potassium supplementation is accompanied by natriuresis. Hypertensive SS patients have less kallikrein excretion; Afro-Americans present a higher prevalence of $\mathrm{SS}$, lower intake of $\mathrm{K}$ and urinary kallikrein, and greater reduction in pressure with $\mathrm{K}$ supplementation. Renal kallikrein stimulation by $\mathrm{K}$ is possibly involved in the greater blood pressure drop that is achieved by decreasing the Na ratio. $\mathrm{K}$, than obtained only by reducing sodium. ${ }^{13}$

Of proinflammatory cytokines such as NF-kB and TNF-a and hypertension in salt-sensitive Dahl rats, and 2) immunosuppression improves salt-sensitive hypertension and increased intrarenal Angiotensin II, probably due to tubulo-interstitial inflammation. In addition, a high sodium intake increases oxidative stress through NAD (P) H oxidase, inhibiting the transport of L-arginine and nitric oxide synthetases. ${ }^{14}$

\section{Clinical manifestations of arterial hypertension (HT)}

Uncomplicated essential hypertension clinic: Mild hypertension, without target organ involvement, is usually totally asymptomatic and its diagnosis is accidental. Within the symptomatology attributable to arterial hypertension, the most constant symptom is headache, but it is more so in those who know the diagnosis than in those who have the same BP level but are unaware that they are hypertensive. The headache is usually fronto-occipital and sometimes awakens the patient in the early hours of the morning. In severe hypertension, the occipital cephalon is more constant and one of the first symptoms to alert the patient. Other symptoms attributed to hypertension, such as ringing in the ears, epistaxis, or dizziness, are not more frequent than in normotensive subjects. ${ }^{15}$

\section{Clinic of complicated arterial hypertension}

The repercussions at the systemic level will be the determinants of the symptoms in these patients; thus, at the cardiac level, symptoms derived from ventricular hypertrophy may appearleft and congestive heart failure and if there is arteriosclerosis of the coronary vessels it will determine ischemic heart disease.

Complications at the central nervous system level can range from a transient ischemic attack to a cerebral infarction in the internal carotid or basilar vertebral territories, lagoon infarcts that, together, can lead to vascular dementia and cerebral hemorrhage, the main etiological factor of which is the arterial hypertension. ${ }^{15}$

\section{Secondary arterial hypertension}

The identifiable causes of arterial hypertension are many, however they are only a cause of hypertension in 5\% of patients, being unknown in $95 \%$ of cases and classified as primary or essential hypertension. 1.6.1. Drug and Drug Induced Hypertension: It is the first cause of secondary hypertension. It is estimated that they account for $30 \%$ of secondary hypertensions: steroid substances11-DOCA. Hormonal contraceptives. Substances with adrenergic activity. Cyclosporine. NSAIDs: They raise blood pressure in hypertensive patients and block hypotensive activity (of ACEI, ARA II) by inhibiting the synthesis of vasodilator prostaglandins (PGE2).

\section{Endocrine-caused hypertension}

Pheochromocytoma: It is estimated that it is a cause of secondary arterial hypertension in $0.02 \%$. Its hypertensive action is due to the production of catecholamines, the clinical manifestations being very varied: paroxysmal crises that appear in more than $75 \%$ of patients and are characterized by headache, sweating and palpitations, as the most frequent symptoms. The crisis is followed by great weakness and sometimes polyuria.

The onset of the crisis can be triggered by pressure in the tumor area, postural changes, exercise, anxiety, pain, urination, etc. Other symptoms may be weight loss, painful hematuria, abdominal, bladder, and / or lumbar pain. The most frequent location is at the adrenal level, in $90 \%$ of the cases, although it is not the only one since it can be found in the mediastinum, at the paravesical level or in the organ of Zuckerkandl.

Pheochromocytomas can be familial in $10 \%$ of cases, inheritance is autosomal dominant. About $60 \%$ of family cases are not accompanied by other endocrine disorders and occurs in children under 20 years of age. Pheochromocytomas or multiple paragangliomas are more common in young women, associated with familial diseases with autosomal dominant inheritance, such as Von Hippel-Lindau disease (cerebral hemangioblastoma), multiple endocrine neoplasia type 2 (MEN-2), and multiple hemangiomas.

Diagnosis is made by determining vanilmandelic acid, total catecholamines, or metanephrines in 24-hour urine. Although they have a high specificity, the sensitivity is $80 \%$. Its location can be obtained specifically by metaiodobenzyl guanidine scintigraphy, which is a radio-tracer with specific uptake by chromaffin tissue. Treatment is surgical, previously alpha-blocking the patient to avoid the discharge of catecholamines during anesthesia or manipulation of the tumor.

a. Hyperthyroidism: hypertension occurs in $30 \%$ of hyperthyroid patients, mainly due to increased cardiac output and blood volume. It is more common in adenomas than in Graves disease.

b. Hypothyroidism: HT occurs in $20 \%$ of patients, mainly due to increased resistance.

c. Hyperparathyroidism: HT is a frequent occurrence (40-70\% of cases) and $40 \%$ have elevated renin values. 
d. Hyperaldosteronism: Aldosterone increases the number of open sodium channels in the luminal part of the collecting duct cells, increasing sodium reabsorption, which creates a negative electrical gradient that favors the secretion of potassium and hydrogen ions. The increase in extravascular volume suppresses renin release, which will be essential for making the differential diagnosis between primary and secondary hyperaldosteronism. The most important symptoms of these situations are muscle weakness, paresthesia and cramps. At the metabolic level, hypokalemia may be absent although it is frequent, metabolic alkalosis, etc. ${ }^{15}$

\section{Materials and methods}

\section{Location}

The present research work was carried out in the environments of the Laboratory of Physiology and Natural Sciences of the UNSCH and in the high Andean localities of the province of Huamanga.

\section{Kind of investigation}

Basic - descriptive.

\section{Research design}

Cross.

\section{Population}

The population of the province of Huamanga.

\section{Show}

It was made up of 110 residents of the high Andean communities such as Huascahura, Rancha, Socos, Vinchos, Quinua, Acosvinchos, Acocro and Mollepata, among others from the province of Huamanga.

\section{Inclusion criteria}

All patients of both sexes from 40 to 70 years of age, who voluntarily decided to take part in the home measurement of blood pressure with symptoms of hypertension.

\section{Exclusion criterion}

Patients of both sexes under 40 and those over 70years of age, who voluntarily decided to take part in the home measurement of blood pressure without symptoms of hypertension.

\section{Methodology}

\section{Barochiner's mdhta diagnostic methods ${ }^{12}$}

Home blood pressure measurement (MDPA) also offers advantages over other ambulatory blood pressure (BP) measurement methods, such as greater acceptance by patients, lower cost and the possibility of monitoring treatment long-term.

1. Preparation of the voluntary consent form.

2. Application of the data collection instrument on dietary salt consumption.

3. The home blood pressure measurement was carried out in the morning hours after coordination with the inhabitants of the high Andean communities and in some cases in the Physiology and Natural Sciences laboratory, free of environmental noise to avoid the tranquility of the inhabitants.

4. Blood pressure values will be obtained in $\mathrm{mmHg}$ and recorded data regarding the pathophysiological effect.

\section{Results}

(Figures 1-3), (Tables 1-3).

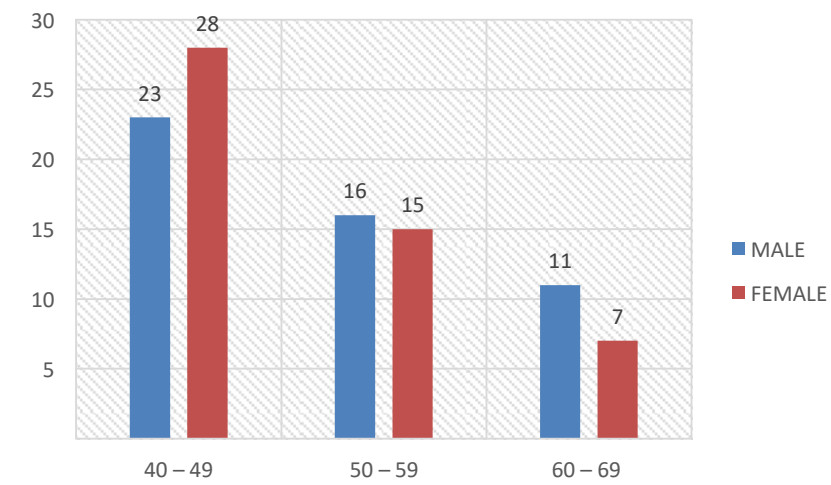

Figure I Age and sex distribution of patients with home blood pressure measurement in the high Andean communities of the province of Huamanga.

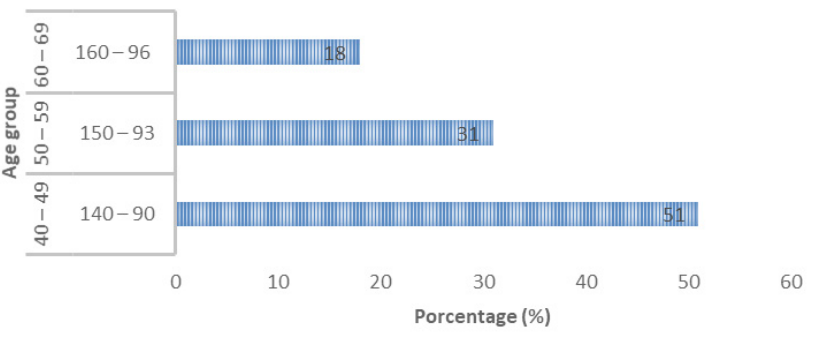

Figure 2 Distribution of home blood pressure measurement according to the age of the high Andean inhabitants of the province of Huamanga.

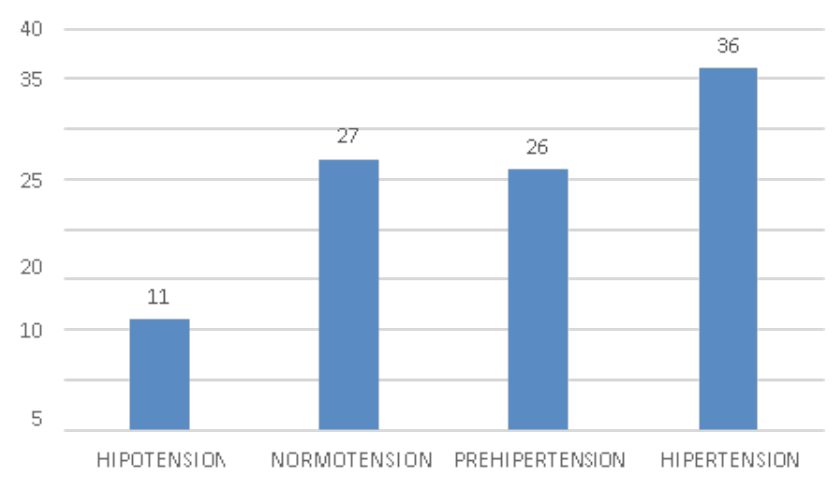

Figure 3 Classification of arterial hypertension in relation to the amount of salt consumed by the high Andean population of the province of Huamanga.

Table I Distribution of the possible pathophysiological effects of hypernatremia in high Andean inhabitants of the province of Huamanga

\begin{tabular}{lll}
\hline Tiphe Phatofisiology & $\mathbf{N}^{\circ}$ & $\%$ \\
\hline Smoking & 13 & 12 \\
Obesity & 10 & 9 \\
Diabetes & 6 & 6 \\
Alcoholism & 15 & 14 \\
Phísical activity & 5 & 4 \\
Nepropatias & 18 & 16 \\
Hearth disease & 8 & 7 \\
\hline
\end{tabular}


Table Continued...

\begin{tabular}{lll}
\hline Tiphe Phatofisiology & $\mathbf{N}^{\circ}$ & $\%$ \\
\hline Polyhydrosis & 10 & 9 \\
Crams and mialgia & 15 & 14 \\
Headaches & 10 & 9 \\
Deaht & 0 & 0 \\
Totals & 110 & 100 \\
\hline
\end{tabular}

Table 2 Distribution of the possible causes of hypernatremia in high Andean inhabitants of the province of Huamanga

\begin{tabular}{lll}
\hline Causas of HTN & $\mathbf{N}$ & $\%$ \\
\hline Drinks & 15 & 14 \\
Deshidration & 21 & 19 \\
Powers & 38 & 35 \\
Diseases & 26 & 23 \\
Sel medication & 10 & 9 \\
Totals & 110 & 100
\end{tabular}

Table 3 Distribution of localities where home care of blood pressure and diagnosis of hypernatremia were performed in high Andean residents of the province of Huamanga

\begin{tabular}{lll}
\hline Localities & $\mathbf{N}^{\circ}$ & $\%$ \\
\hline Huascahura & 15 & 14 \\
Rancha & 20 & 18 \\
Socos & 8 & 7 \\
Vinchos & 6 & 5 \\
Quinua & 25 & 24 \\
Acosvinchos & 20 & 18 \\
Acocro & 6 & 5 \\
Mollepata & 10 & 9 \\
Total & 110 & 100 \\
\hline
\end{tabular}

\section{Discussion}

Figure 1 shows a general distribution of age and sex of patients with home blood pressure measurement in the high Andean communities of the province of Huamanga, where a lower percentage $(39 \%)$ corresponds to the male sex and in the highest proportion were female $(61 \%)$. For the age group of 40 to 49 years there is a greater predominance of the female sex with $28 \%$ and the male sex with $23 \%$, for the other age groups of 50 to 59 years and from 60 to 69 years the male sex predominates with a slight percentage.

In other words, in marginal urban and rural areas there are more adults and the elderly, due to socio-political factors that affected the Ayacucho region. In the consultations carried out, we were able to observe that in a good part of them they are the survivors or were abandoned by their relatives when they migrated to other areas in search of refuge or went in search of work, and for many reasons they no longer returned, leaving to their fate to their elderly parents, despite everything and over the years they still carry out some agricultural or grazing work, they said that they had almost never seen this type of action in their place and if they go to the post it is only due to some ailment that medicinal plants cannot heal.

Regarding Figure 2, we note the distribution of the home blood pressure measurement according to the age of the high Andean inhabitants of the province of Huamanga, that 110 people attended with ages between 40 and 49 years of age obtained a mean of 140 to $90 \mathrm{mmHg}$ of blood pressure, constituting $51 \%$ followed by people with ages of 50 to 59 years with 150 to $93 \mathrm{mmHg}$ of blood pressure, constituting $31 \%$ and in third place people with ages between 60 to 69 years of $16096 \mathrm{mmHg}$ corresponding to $18 \%$ which shows that children and young people go to study in the city, some of them are not used to the way of living in the countryside, only the adults are cultivating, herding and caring for their humble abode. In other words, there is a small percentage of adults who are farmers or small ranchers or animal shepherds, who stayed to form their new family and dedicate themselves to work corresponding to the subsistence of their progeny, they do not migrate to the city because they do not have relatives, thus they manifest it.

In Figure 3. We observe the classification of arterial hypertension in relation to the amount of salt consumed by the high Andean population of the province of Huamanga, according to the amount of salt consumed. $11 \%$ of hypotensive patients consume less than 4 grams of salt, normotensive patients use 5 grams of salt, with a percentage of $27 \%$ that manifest normal consumption as indicated by the WHO, while pre-hypertensive patients consume 7 grams of salt correspond to the $26 \%$ but in a greater proportion we find people who consume more than 10 grams of salt with $36 \%$ this makes us see that the high Andean population likes a lot of salt and they even made us try a sample of their food, including the very salty cheese, Much consumed in the rural population along with fried or parboiled potatoes, corn roasted with salt, other residents even say they add more salt when their food does not have a taste like the fruits of caigua, avocado and other fruits. It is more to observe in the patio of their houses drying the salted meat or jerky as a way to preserve their food.

It is clear that salt, and the handling of this ion by the kidney, are the most important cause of increased blood pressure. There are so many ways to retain sodium (Na), the most logical thing is to think that the sensitivity to salt is determined by different genotypes, acting in a monogenic way in small groups of hypertensive patients, and in polygenic combinations that interact in the majority with the phenotype (sex, age, obesity, reduced kidney function).

These genotypes would have accumulated throughout evolution to allow survival in low supply conditions, but at present they present a significant risk for developing hypertension. Even though so far we cannot identify sensitive individuals with simple interventions, the proven benefit of a moderate restriction and the non-pressurizing effects of salt amply justify the recommendation of an intake between 3.5 and $6 g$ per day.

Table 1 shows the distribution of the pathophysiological effect of hypernatremia in high Andean inhabitants of the province of Huamanga, with possible pathophysiological alterations, as indicated by the inhabitants, they present pain or have suffered the effects of these pathologies, with a higher percentage of alcoholism $15 \%$, nephropathy $18 \%$ and cramps and myalgia with $15 \%$, while the pathologies with a similar percentage are obesity, polyhydrosis and headaches with $10 \%$ and to a lesser extent we have $13 \%$ smoking, $6 \%$ diabetes, lack of physical activity $5 \%$, heart disease $8 \%$ and there were no deaths $0 \%$. 
In Table 2 on the distribution of the possible causes of hypernatremia in high Andean inhabitants of the province of Huamanga, according to the answers given by the inhabitants the possible causes that generate hypernatremia, a list of possible causes is shown between They have the following, $14 \%$ say they have consumed drinks mixed with blue salt or soaked, $19 \%$ is due to dehydration suffered when carrying out agricultural work for several hours and hot days, $35 \%$ state that it is due to food consumption very salty, they generally accompany the salt in various products as additives to preserve their meat or cheese foods and in this way prevent their deterioration or decomposition and contamination of insects, especially against flies and other rodents. $23 \%$ indicate that it may be due to having suffered some diseases such as obesity, alcoholism, kidney disease, diabetes and other pathologies such as excessive consumption of fat in the form of sebum, fried foods, pachamancas and chicharrones, when they sacrifice their animals raised by them. $9 \%$ report having self-medicated with cortisol-based products or another cortisone used when treating these pathologies.

Obesity is positively correlated with salt reabsorption in the proximal tubule. Among the mechanisms that explain this greater reabsorption are the activation of the renin-angiotensin system and the adrenergic system, hyperinsulinemia, and morphological changes (mesangial fibrosis, spinal compression due to an increase in the interstitial matrix. ${ }^{15}$

Finally we have Table 6 , referring to the distribution of localities where home care of blood pressure and the diagnosis of hypernatremia were performed in high Andean inhabitants of the province of Huamanga, they were in higher percentage in Huascahura, Rancha, Quinua, Acosvinchos and the towns with the least number of people served were Socos, Vinchos, Acocro and Mollepata, it should be noted that it is not the lack of will of the undersigned and his assistants, but rather the idiosyncrasy of the population by not wanting to collaborate, we must respect their beliefs. Also note that the list of locations was related to the order of visits made.

The prevalence of hypertension in a population or, in other words, the proportion of individuals who are hypertensive at a given time, varies widely depending on certain factors, which basically include the age group of the population considered, the methodology used to measure blood pressure, the number of readings taken and, above all, the limit chosen to differentiate normotension from hypertension. This last factor is the most determining factor when estimating prevalence. Indeed, whether the classic limit of $160 / 95 \mathrm{mmHg}$ or the more current $140 / 90 \mathrm{mmHg}$ is chosen definitely determines the prevalence of hypertension in the population. On an international scale, using the $160 / 95 \mathrm{mmHg}$ limits, the registered prevalences range from 10 to $20 \%$ of the adult population (18years and over). If the limits used are $140 / 90 \mathrm{mmHg}$, the prevalence can increase to $30 \%{ }^{15}$

\section{Conflicts of interest}

No conflict of interest.

\section{Funding}

None.

\section{References}

1. Parati et al. European Society of Hypertension Practice Guidelines for Home blood pressure monitoring. 2010;24 (12):779-85.

2. Pickering et al. Action on Use and Reimbursement for Home Blood Pressure Monitoring. Hypertension. 2008;52:10-29.

3. Niiranen et al. Home-measured blood pressure is a stronger predictor of cardiovascular risk as office blood pressure: the Finn Home study. Hypertension. 2010;55:1346-1351.

4. Alderman M. A pinch of science. Opinion article. New York Times, November 6, 2014. USA.

5. Herrera, J. Salt-dependent hypertension. Edit. INC. Nephrology Department. 2008. Mexico.

6. Weinberger M, Fineberg N. Sodium and volume sensitivity of blood pressure. Age and pressure change over time. Hypertension. 1991;18:6771.

7. Guyton et al. Arterial pressure regulation. Overriding dominance of the kidneys in long-term regulation and in hypertension. Am J Med USA. 1972;52:584-594.

8. Lombardi et al. Salt-sensitive hypertension develops after short term exposure to angiotensin II. Hypertension.1999;33:1013-1019.

9. Sommers SM, Relman R, Smithwick R. Histologic studies of kidney biopsy specimens from patients with hypertension. Am $J$ Pathol. 2008:34:685-715

10. Sanchez R, Ramirez A. Arterial hypertension and hypersensitivity to salt 7th Virtual Congress of Cardiology. 2011. USA.

11. Juncos et al. Importance of sodium and high blood pressure. Rev. Arterial hypertension. National University of Cordoba. 2012. Argentina.

12. Barochiner J. Reproducibility and reliability of a 4-day LMWH protocol with and without first day measurements. Journal of the Faculty of Medical Sciences of the National University of Córdob. 2011;68 (4):149-153.

13. Valdés G. Salt and arterial hypertension. Rev. Human Medicine. Pontifical Catholic University. 2009. Santiago.

14. Orias M. Sodium concentration and blood pressure. Rev. HTA. 2010. Córdova.

15. Castell E. Arterial hypertension. Rev. Internal Medicine. University Clinical Hospital of Malaga. 2010. Spain.

\section{Acknowledgments}

None. 